\title{
Healing of toothbrush-induced abrasions to keratinized mucosa of the palate in humans: A pilot study.
}

Mathieu De Nutte $\S^{\star}$, Elisabeth Behaeghe ${ }^{\S^{*}}$, Fridus van der Weijden\#, Wim Coucke ${ }^{\S}$, Wim Teughels§ \& Marc Quirynen§

$\S$ Department of Oral Health Sciences, KU Leuven \& Dentistry, University Hospitals Leuven, Kapucijnenvoer 33, 3000 Leuven, Belgium

\# Department of Periodontology, Academic Center for Dentistry Amsterdam, University of Amsterdam and Vrije Universiteit, Amsterdam, The Netherlands

* Both the authors have contributed equally to the work

\section{Correspondence to:}

Mathieu De Nutte

Catholic University Leuven,

Department of Periodontology

Kapucijnenvoer 33,

3000 Leuven, Belgium

Tel: +32.16 .33 .24 .83$

Email: mathieu.denutte@hotmail.com

Fax: +32.16.33.24.84

Short title: Healing of gingival abrasion.

Source of funding: The study was self-funded by the authors and their institution.

Conflict of interest: The authors have stated explicitly that there are no conflicts of interest in connection with this article.

Summary: Cumulative damage is feasible in case of repeated trauma concerning a lesion that has not adequately healed, which may eventually lead to more serious adverse results such as gingival recession.

Key-words: gingival diseases, gingival recession, soft tissue injuries, gingiva, gingivitis, oral hygiene, dental plaque, tooth brushing, wound healing 


\section{Abstract}

Objective \& Background: To our knowledge, the healing time for toothbrush-induced gingival abrasions in humans has not yet been explored. The aim of this study was to evaluate the time needed for toothbrush-induced abrasions of the keratinized mucosa of the palate, to heal.

Materials \& Methods: In patients without, with mild periodontitis or with periodontitis, gingival abrasion lesions were induced at the keratinised mucosa of the palate by brushing up to 2 minutes. Healing as observed clinically was followed via calibrated digital photographs of the lesion after staining, every 24 hours until the lesion had resolved. In patients without or with only mild periodontitis, the healing time of lesions caused by 30 seconds and 1 minute of brushing was also recorded.

Results: 31 participants (11 without or with mild periodontitis, 20 with periodontitis) met the inclusion criteria. In patients without or with mild periodontitis, lesions induced by brushing for 2 minutes, diminished by $48.7 \%$ of their original surface area within 24 hours. In patients with periodontitis the respective outcome was $45.4 \%(p=0.87)$. Abrasions caused by 2 minutes of brushing needed more than 24 hours to heal completely. When decreasing the time exposed to trauma, fewer lesions were visible at baseline and the time needed to heal decreased. The subgroup of smokers was too small to evaluate any effect.

Conclusion: The longer the exposure time of the keratinized mucosa of the palate to trauma, the more lesions were visible, the larger the abrasions were and the more time they needed to heal completely. More studies are needed to investigate the role of smoking, gingival biotype and to link these findings with gingival recessions. 


\section{Introduction}

As observed in epidemiological studies, gingival recessions are common among adults $^{1,3,4,13,20,27}$ and can be localized or generalized. The prevalence, extension and severity of gingival recession gradually increases with age $\mathrm{e}^{1,13,27}$. This relation can be explained by the longer period of exposure to an etiological cause for gingival recession and the cumulative effect of this rather than aging itself 11,20 .

The development of recession is believed to be multifactorial, including anatomical and pathological factors, orthodontic movement and various forms of trauma ${ }^{1,10}$. One of the main causes of gingival recessions is inflammatory plaque-induced periodontitis ${ }^{24}$. Other possible causes are orthodontic treatment and trauma from tooth brushing. The latter has been suggested as a necessary cause for gingival recession in various studies $^{9,11,22}$. In this pilot study, we will focus on the non-plaque induced gingival recessions/abrasions, more specifically toothbrush-related trauma to the soft tissues. It has been shown that the use of hard-bristled toothbrushes ${ }^{11}$ or toothbrushes with cut bristle ends ${ }^{2}$ can increase the risk of gingival trauma. Some studies indicate that also the brushing technique plays a significant role ${ }^{17}$ while others show that patients who brush their teeth more frequently have more recessions ${ }^{3,11,27}$. Patients with a high standard of oral hygiene tend to have more gingival recessions, especially on the buccal surfaces ${ }^{20}$.

However, as concluded in a systematic review the data to support or refute the association between tooth brushing and gingival recession were considered to be inconclusive ${ }^{17}$. Of the 17 studies included, only 8 reported a positive association between tooth brushing frequency and recession. Other potential risk factors were 
duration of tooth brushing, brushing force, frequency of changing the toothbrush, brush (bristle) hardness and tooth brushing technique.

The exact mechanism by which tooth brushing can lead to gingival recession is at present still not fully understood. It has been suggested that toothbrush-associated trauma of the soft tissues, by either manual or powered tooth brushing could contribute to its development ${ }^{18,26}$. Toothbrush-associated trauma can be clinically observed as gingival abrasion lesions or gingival fissures ${ }^{8}$. Several studies investigated the prevalence of gingival abrasion lesions and fissures in association with tooth brushing and found that they are common $6,8,18,19$. Gingival abrasion is a superficial damage to the keratinized epithelial layer of the gingival tissues. It is commonly expected that these lesions will heal uneventfully ${ }^{16}$. To our knowledge no study has yet investigated the healing time of such abrasions in humans. Additionally, the question to what extent these superficial damages are associated with gingival recession remains open because the direct relation between traumatic home care and gingival recession is inconclusive $e^{12,17,18}$.

Therefore, the aim of the present study was to evaluate the healing time of a lesion caused by brushing in order to investigate if cumulative damage is possible. Thus, if the patient causes trauma to the keratinized mucosa by brushing his teeth and this lesion could not heal completely before a second trauma is caused, additional and probably more extensive damage might occur (cumulative damage). In addition, the hypothesis of differences in healing time between patients with and without periodontitis was tested in order to explore if inflammation of the gingiva has a negative influence on healing. 


\section{Material and Methods}

\section{Study Population}

This pilot study took place at the department of Periodontology of the University Hospitals Leuven, Belgium. Two groups of participants were enrolled.

The first group consisted of volunteers with no or mild periodontal diseases, but without any recessions. They were recruited from employees working at the dental clinic. They received an oral examination and the Dutch Periodontal Screening Index (DPSI) was recorded ${ }^{14,25}$. The main inclusion criterion for this group was DPSI $\leq 3-$, which corresponds with a maximum probing depth of $4-5 \mathrm{~mm}$ in absence of gingival recessions. In what follows, this group will be referred to as that of the non-periodontitis patients.

The second group consisted of patients with active periodontitis, recruited during their first consultation. For this group, the requirement was DPSI of 4, indicating at least one pocket with a probing depth (PPD) of $6 \mathrm{~mm}$ or more. The additional inclusion and exclusion criteria are summarised in Table 1.

Gender, age, smoking status and gingival biotype were recorded for all participants. Gingival biotype was recorded visually and by using a periodontal probe. Gingival biotypes were classified into three categories according to the classification described by De Rouck and co-workers in $2009^{7}$. Thin, thick flat and thick scalloped gingival biotypes could be distinguished.

The study was conducted in accordance with the ethical principles in the Declaration of Helsinki and consistent with Good Clinical Practice guidelines. The protocol was approved by the Medical Ethics Committee of the University Hospitals Leuven 
(B322201523818). Before the start of the study, participants were informed about the purpose, procedures and duration of the study, and informed consent was obtained

\section{Study design}

Figure 1 presents the flowchart of the study.

\section{Part 1: 2 minutes of brushing}

At baseline the examiner brushed the gingiva of the palate for two minutes to induce gingival abrasion in this area. Gingival abrasions were obtained by making small circles with a conventional multi-tufted medium-hard toothbrush (Sensodyne®) (Figure 2). Due to the curvature of the palate, particularly the tip of the toothbrush was in contact with the palate. The number of rotations per minute was not fixed, but variation in applied forces was kept to a minimum because it was always the same clinician who introduced the lesions, trying to follow the same protocol. This was performed in every patient of both groups by the same clinician. The brushing area was not in contact with the gingival margin to prevent lesions at the gingival margin, which could lead to gingival recession. After inducing the abrasion lesion, the palatal gingiva was stained for 30 seconds (Mira-2-Ton® Hager and Werken, GMBh \& Co., Duisburg, Germany). The stain visualised the areas where the surface of the oral epithelium had been damaged (blue discoloration). This technique was first introduced by Breitenmoser and co-workers ${ }^{2}$ and adapted by Danser and co-workers ${ }^{6}$. After staining, clinical pictures were taken (as parallel to the palate as possible) with a ruler (calibrated in $\mathrm{mm}$ ) next to the abrasion (Figure 2). This procedure was repeated daily, until complete resolution of the lesions. The participants were asked not to use any mouthwashes during the experimental period. No other restrictions were given. 


\section{Part 2: 30 seconds \& 1 minute of brushing}

After a break of at least 2 weeks, the non-periodontitis group was invited to participate in a second experiment. The same protocol as the one described above was used but this time the examiner brushed the palate on the right side for 1 minute and that on the left side for 30 seconds. The follow-up of this experimental period was conducted in the same manner until the abrasions were no longer visible.

\section{Abrasion surface area}

The surface area of each lesion was calculated using the PictZarß-PRO ver.7.5.0 BR computer programme. The same examiner who had performed the clinical examinations did the calculations. For each patient, the images of the lesions were randomized as to the time points they were created and the time period needed for them to heal. To determine the intra-examiner correlation, 12 lesions were scored randomly a second time by the same examiner one month after the first measurements to reduce the risk of bias. The intra-examiner correlation was $0.99(\mathrm{Cl}$ [0.993; 0.999)].

\section{Statistical analyses}

For categorical variables frequency tables were created. For numeric variables, the following values were calculated: mean, standard deviation, and the first, second and third quartile. Pearson correlation coefficients and their corresponding P-value were calculated to establish the relation between lesion sizes and brushing times. The initial wound area was $\log ^{10}$ - transformed to make the distribution in the regression analysis less skewed and to make the pattern more interpretable. 


\section{Results}

\section{Patient demographics}

A total of 31 volunteers met the inclusion criteria and were willing to participate. The 11 participants in the non-periodontitis group had a mean age of 24.2 years (range 2326). Nine of them were women. The periodontitis group $(n=20)$ had a mean age of 48.2 years (range 30-73) and included 14 women. There were 3 smokers in the periodontitis group and none in the non-periodontitis group. During the course of the study none of the participants withdrew from the study. The demographic characteristics of the participants are summarized in Table 2.

\section{2 minutes of brushing}

Table 3 shows the average abrasion surface area $\left(\mathrm{mm}^{2}\right)$ caused by 2 minutes of brushing per 24 hours' interval for both groups. At baseline the mean surface area of the abrasion lesion was $22.5 \mathrm{~mm}^{2}( \pm 24.7)$ in the non-periodontitis group and $25.5 \mathrm{~mm}^{2}$ $( \pm 34.8)$ in the periodontitis group. In the latter group, no visible lesion could be induced in 5 participants. Therefore, they were excluded from the analysis.

After 24 hours of healing, the abrasion lesions had a mean surface area of $12.3 \mathrm{~mm}^{2}$ $( \pm 12.3)$ in the non-periodontitis group and $18.3 \mathrm{~mm}^{2}( \pm 24.8)$ in the periodontitis group. This corresponds with an area reduction of $48.7 \%$ in the non-periodontitis group versus $45.4 \%$ in the periodontitis group during these first 24 hours $(p=0.87)$. Only in one participant of the non-periodontitis group and in 2 subjects of the periodontitis group, did the lesions not show staining within 24 hours. These lesions had an initial size of $0.8,6.5$ and $3.3 \mathrm{~mm}^{2}$, respectively.

After 48 hours of healing the mean surface area of the lesions decreased to $6.2 \mathrm{~mm}^{2}$ $( \pm 9.6)$ in the non-periodontitis group, with lesions still visible in 7 out of 11 participants. 
In the periodontitis group, the average surface area decreased to $17.1 \mathrm{~mm}^{2}( \pm 22.0)$. In this group, the lesion was still visible in 6 out of 16 participants.

After 72 hours, most lesions were no longer visible. In the non-periodontitis group, 3 lesions remained and their mean surface area was reduced to $8.4 \mathrm{~mm}^{2}( \pm 12.6)$. In the periodontitis group only 4 lesions were still visible with a mean surface area of 12.6 $\mathrm{mm}^{2}( \pm 13.0)$.

After 96 hours, all but one lesion had resolved in the non-periodontitis group. This lesion remained visible for another 5 days. In the group with periodontitis, 1 lesion remained visible after 96 hours and for 8 days. These lesions had an initial surface area of 78.2 and $88.2 \mathrm{~mm}^{2}$, respectively.

\section{1 minute \& 30 seconds of brushing}

In the non-periodontitis group, abrasion lesions were also induced by brushing for 1 minute and 30 seconds. Table 4 shows the average abrasion surface area after 24 hour intervals of healing. In six participants, a lesion was obtained after 1 minute of brushing and after 30 seconds only in 5 participants. The abrasion lesions had a mean surface area of $7.4 \mathrm{~mm}^{2}( \pm 9.0)$ and $1.4 \mathrm{~mm}^{2}( \pm 0.9)$ after 1 minute and 30 seconds, respectively.

The mean surface area after 24 hours of healing was $4.0 \mathrm{~mm}^{2}( \pm 2.7)$ and $0.5 \mathrm{~mm}^{2}( \pm$ 0.4 ) for lesions caused by 1 minute and 30 seconds of brushing, which represents a reduction of 43.1 and $73.9 \%$, respectively. After 24 hours, 5 out of 7 lesions caused by 1 minute of brushing remained. The lesions caused by 30 seconds of brushing were still visible in 2 out of 5 participants.

After 48 hours of healing only 1 lesion caused by 1 minute of brushing remained, and all lesions caused by 30 seconds of brushing, were resolved. 


\section{Susceptibility to abrasion}

A Pearson correlation was used to analyse the relation between the surface areas of lesions caused by variable timing ( 2 minutes, 1 minute, 30 seconds). A significant correlation $(0.74, p=0.01)$ was found between the surface area caused by 2 and 1 minute of brushing and between 1 minute and 30 seconds of brushing $(0.71, p=0.02)$. The correlation between the 2 minutes and 30 seconds' lesions was weak $(0.44$, $\mathrm{p}=0.18)$

\section{Healing time}

Figure 3 shows the relation between the initial abrasion area and the healing time. The healing time of abrasion lesions for both non-periodontitis and periodontitis patients was significantly related to the initial abrasion area $(p<0.001)$. However, the slopes of the two respective regression lines were statistically non-significant $(0.272$ for nonperiodontitis patients vs. 0.369 for periodontitis patients $)(p=0.40)$. 


\section{Discussion}

This study was conducted in an effort to investigate the time needed for toothbrushassociated abrasion lesions to the keratinized mucosa of the palate to be induced and to resolve. The results show that an average abrasion lesion caused by 2 and 1 minute of brushing needed more than 24 hours to heal in most of the cases. An average abrasion lesion caused by 30 seconds of brushing needed at least 24 hours to heal in $40 \%$ of the cases. This suggests that cumulative damage is possible if a new trauma from a new brushing exercise is introduced within the first 24 hours. This finding corresponds with the general assumption/hypothesis in the literature that gingival recession could be the result, among other factors, of cumulative damage over a prolonged period of time $e^{8,11,20}$. The possible link between traumatic tooth brushing and gingival recession is extensively discussed in the literature. In 1979 Breitenmoser and co-workers ${ }^{2}$ already suggested that repeated traumatic tooth brushing could lead to irreversible gingival defects in the form of recession. On the other hand, two reviews found the relation between gingival abrasion and recession to be inconclusive ${ }^{12,17}$. Furthermore, a recent cross-sectional study in which manual and powered toothbrushes were evaluated also could not show a correlation between toothbrushassociated gingival abrasion and gingival recession ${ }^{18}$. This study compared the number of sites with gingival abrasion pre-brushing to the number of sites with gingival abrasion post-brushing. The incremental change, which was considered as representative for the amount of 'trauma' an individual could induce, showed no correlation with the presence of recessions. The population in this study consisted of periodontally healthy individuals and the prevalence of recession was low. Altogether, no clear-cut conclusion could be drawn regarding cumulative trauma from brushing because there were no follow-up studies on the healing of such defects. 
There is a scarcity in long-term follow-up studies investigating the chronic effect of abrasion damage. Smith ${ }^{21}$ proposed that recessions are the consequence of chronic trauma which induces a chronic inflammatory response in the tissues followed by the destruction of the underlying bone with recession as the endpoint. A recent study found a relation between traumatic tooth brushing and the occurrence of gingival fissures during a 28-day observation period ${ }^{8}$. Another study, with a 12-month follow-up period, which investigated the impact of a normal oral hygiene on the onset and/or progression of gingival recession, found no correlation ${ }^{15}$. Gingival abrasion and fissures can be the clinical result of a trauma to the soft tissues. Apart from tooth brushing appearing to be a necessary cause, more is needed to develop gingival recession. It would be interesting to investigate to what extent the soft tissues must be damaged before the underlying bone is irritated, disappears and recession becomes apparent.

The present study was limited in that it did not include power analysis, identical groups regarding mean age, number of smokers.

In this pilot study staining was used to visualize the gingival abrasion lesion. However, within the limits of the present study, it was impossible to determine to what extent the gingiva was damaged. Furthermore, the present study considered a lesion to have healed if it no longer displayed the blue stain at the next assessment. However, it was impossible to verify whether structure and thickness of the epithelium were fully recovered when it was considered healed after applying the disclosing agent.

An abrasion lesion could not be induced in every participant (5/20), not even by 2 minutes of brushing. This was also observed in the group where the lesions were induced by 1 minute and 30 seconds of brushing. This is in agreement with the general 
knowledge that not everyone is equally sensitive to abrasion lesions and gingival recession ${ }^{12}$.

In the non-periodontitis group, lesions were induced 3 times. In this group, it was observed that the possibility to induce a lesion was patient-dependent. In some patients, no lesion could be induced, neither after 30 seconds nor after 1 minute of brushing. In the same patients, the lesions after 2 minutes of brushing healed faster. Other patients showed longer healing times for all lesions induced by different brushing times. The gingival biotype seems to play a significant role $(p<0.05)$. Abrasion lesions could be more easily induced in patients with a thin (scalloped) biotype, and in these patients the lesions showed longer healing times.

The present study investigated the healing time of a freshly induced abrasion lesion. An area away from the gingival margin at the palate was chosen as experimental area because no toothbrush-associated abrasion lesions could be present at baseline. On the other hand, a lesion-free palate was rare. Prior lesions may have been the result of the consumption of hard or hot food. Differential diagnosis between such lesions and toothbrush-associated abrasion lesions was made based on their location verified on the consecutive pictures. A second reason to choose the palate was the necessity to stay away from gingival margins where these experimentally induced lesions could have resulted in deleterious changes of the gingival margin. As a consequence, one should take into account that the soft tissue at the palate is not comparable with that at the gingival margin, especially at the gingival margin of patients with periodontitis, where more vascularisation, more inflammatory cells, wider intercellular spaces, can be observed ${ }^{5,23}$. 
To explore if the healing time is influenced by the periodontal condition of the participants, the present study induced abrasion lesion in both patients with and without periodontitis. The results indicated that there was no significant difference in healing $(p=0.87)$ between both groups. Therefore, one could conclude that periodontal health had no impact on the healing time with an area reduction of $48.7 \%$ in the nonperiodontitis group versus $45.4 \%$ in the periodontitis group during the first 24 hours, respectively. An aspect one should bear in mind is the fact that the lesions were induced to the palate and not to the gingival margin. As a second aspect, there was the difference in mean age of both groups. Young subjects may well show better healing compared to older subjects. Both may have influenced our results. No statistically significant difference between the initial abrasion area and healing time between the periodontitis and non-periodontitis patients was found $(p=0.40)$. In the group with periodontitis, 3 out of 20 patients were smokers. In only 1 of these 3 smokers could an abrasion lesion be induced. This subgroup was too small to evaluate the effect of smoking.

Patients probably never brush for 2 minutes continuously at the same place. Hence, abrasion lesions by brushing 1 minute or 30 second probably simulate a more regular brushing habit. The results of the second experimental period showed that the abrasion lesions caused by 1 minute of brushing still needed $>24$ hours to heal in $5 / 6$ of the cases. Moreover, abrasion caused by 30 seconds of brushing also needed $>24$ hours of healing in $2 / 5$ of cases. Pearson correlation showed that when less time was used for causing the abrasion, the lesions were smaller. The results also showed that the healing time was shorter for the latter (Table 4).

To our knowledge, this is the first paper that examined the healing time for toothbrushassociated abrasion lesions in humans. Histological data on the healing of these 
lesions in dogs showed that gingival abrasions induced by a single brushing period are mostly epithelium lesions with a significant reduction in thickness present up to 24 hours and showing a tendency to recovery after 8 hours of observation ${ }^{16}$. This pilot study provides some insights into the healing of toothbrush-induced trauma at the keratinized mucosa of the palate. This information can be used to set up new clinical trials, taking into account the limitations of this study. Future studies should include a long-term follow-up period to further investigate to what extent and under which circumstances gingival abrasions are involved in the onset and progression of gingival recessions. Furthermore, the influence of smoking in the onset and healing of these gingival abrasions should be further explored in clinical trials with larger groups.

\section{Conclusion}

The data show that the longer the exposure time of the keratinized mucosa of the palate to trauma was, the more abrasion lesions were visible, the larger they were and the more time they needed to heal. Lesions caused by 2 and 1 minute of brushing needed more than 24 hours to show healing in most cases. An average abrasion lesion caused by 30 seconds of brushing needed at least 24 hours to heal in $40 \%$ of cases. This could suggest that cumulative damage is possible if a new trauma from a new brushing exercise is introduced within the first 24 hours. However, more studies are required to confirm this and more clinical trials with a larger number of patients are needed to evaluate the role of smoking, gingival biotype and to link these findings with gingival recessions. 


\section{Acknowledgments}

The authors received no financial support and declare no potential conflicts of interest with respect to the authorship and/or publication of this article. 


\section{References}

1. Albandar JM, Kingman A. Gingival recession, gingival bleeding, and dental calculus in adults 30 years of age and older in the United States, 1988-1994. J Periodontol 1999; 70: 30-43.

2. Breitenmoser J, Mörmann W, Mühlemann HR. Damaging effects of toothbrush bristle end form on gingiva. J Periodontol 1979; 50: 212-216.

3. Checchi L, Daprile G, Gatto MR, Pelliccioni GA. Gingival recession and toothbrushing in an Italian School of Dentistry: a pilot study. J Clin Periodontol 1999; 26: 276-280.

4. Chrysanthakopoulos NA. Prevalence and associated factors of gingival recession in Greek adults. J Investig Clin Dent 2013; 4: 178-185.

5. Ciano J, Beatty BL. Regional Quantitative Histological Variations in Human Oral Mucosa. Anat Rec 2015; 298: 562-578.

6. Danser MM, Timmerman MF, IJzerman $\mathrm{Y}$, Bulthuis $\mathrm{H}$, van der Velden $\mathrm{U}$, van der Weijden GA. Evaluation of the incidence of gingival abrasion as a result of toothbrushing. J Clin Periodontol 1998; 25: 701-706.

7. De Rouck T, Eghbali R, Collys K, De Bruyn H, Cosyn J. The gingival biotype revisited: transparency of the periodontal probe through the gingival margin as a method to discriminate thin from thick gingiva. J Clin Periodontol 2009; 36: 428433.

8. Greggianin BF, Oliveira SC, Haas AN, Oppermann RV. The incidence of gingival fissures associated with toothbrushing: crossover 28-day randomized trial. J Clin Periodontol 2013; 40: 319-326.

9. Joshipura KJ, Kent RL, DePaola PF. Gingival recession: intra-oral distribution and associated factors. J Periodontol 1994; 65: 864-871.

10. Kassab MM, Cohen RE. The etiology and prevalence of gingival recession. J Am Dent Assoc 2003; 134: 220-225.

11. Khocht A, Simon G, Person P, Denepitiya JL. Gingival recession in relation to history of hard toothbrush use. J Periodontol 1993; 64: 900-905.

12. Litonjua LA, Andreana S, Bush PJ, Cohen RE. Toothbrushing and gingival recession. Int Dent J 2003; 53: 67-72.

13. Löe $H$, Anerud $A$, Boysen $H$. The natural history of periodontal disease in man: prevalence, severity, and extent of gingival recession. J Periodontol 1992; 63: 489-495.

14. Mantilla Gómez S, Danser MM, Sipos PM, Rowshani B, van der Velden U, van der Weijden GA. Tongue coating and salivary bacterial counts in healthy/gingivitis subjects and periodontitis patients. J Clin Periodontol 2001; 28: 970-978. 
15. McCracken GI, Heasman L, Stacey F, et al. The impact of powered and manual toothbrushing on incipient gingival recession. J Clin Periodontol 2009; 36: 950957.

16. Oliveira SC, Oppermann RV, Kantorski KZ, Pannuti CM. AVALIAÇÃO HISTOLÓGICA DAS ABRASÕES GENGIVAIS PROMOVIDAS POR ESCOVAÇÃO NO CÃO. Universidade Federal de Santa Maria.

17. Rajapakse PS, McCracken GI, Gwynnett E, Steen ND, Guentsch A, Heasman $P A$. Does tooth brushing influence the development and progression of noninflammatory gingival recession? A systematic review. J Clin Periodontol 2007; 34: 1046-1061.

18. Rosema NAM, Adam R, Grender JM, Van der Sluijs E, Supranoto SC, Van der Weijden GA. Gingival abrasion and recession in manual and oscillating-rotating power brush users. Int J Dent Hyg 2014; 12: 257-266.

19. Sandholm L, Niemi ML, Ainamo J. Identification of soft tissue brushing lesions. A clinical and scanning electron microscopic study. J Clin Periodontol 1982; 9: 397401.

20. Serino G, Wennström JL, Lindhe J, Eneroth L. The prevalence and distribution of gingival recession in subjects with a high standard of oral hygiene. $J$ Clin Periodontol 1994; 21: 57-63.

21. Smith RG. Gingival recession. Reappraisal of an enigmatic condition and a new index for monitoring. J Clin Periodontol 1997; 24: 201-205.

22. Smukler $\mathrm{H}$, Landsberg $\mathrm{J}$. The toothbrush and gingival traumatic injury. $J$ Periodontol 1984; 55: 713-719.

23. Soames JV, Entwisle DN, Davies RM. The Progression of Gingivitis to Periodontitis in the Beagle Dog: a Histological and Morphometric Investigation. $J$ Periodontol 1976; 47: 435-439.

24. Tugnait A, Clerehugh V. Gingival recession-its significance and management. $J$ Dent 2001; 29: 381-394.

25. Van der Velden $U$. The Dutch periodontal screening index validation and its application in The Netherlands. J Clin Periodontol 2009; 36: 1018-1024.

26. Van der Weijden FA, Campbell SL, Dörfer CE, González-Cabezas C, Slot DE. Safety of oscillating-rotating powered brushes compared to manual toothbrushes: a systematic review. J Periodontol 2011; 82: 5-24.

27. Vehkalahti M. Occurrence of gingival recession in adults. J Periodontol 1989; 60: 599-603. 


\section{Figure Legend}

- Figure 1. Study flow chart.

- Figure 2. (a) Gingival abrasion after 2 minutes of brushing (b) Clinical picture with ruler calibrated in $\mathrm{mm}$. All pictures were taken after disclosing with Mira-2Ton®.

- Figure 3. Regression lines for the relation between initial abrasion area and healing time for both groups (non-periodontitis/periodontitis group). The initial abrasion area was $\log ^{10}$-transformed. 


\section{Tables}

Table 1. Inclusion and exclusion criteria

\section{Inclusion criteria}

- Good general health

- $\quad$ Age between 20 and 75 years

- Presence of at least 20 teeth

- Presence of at least 5 teeth per quadrant

\section{Exclusion criteria}

- Unable to provide consent or comply with the study protocol

- Antibiotic intake 6 months before the screening visit

- Treatment with antibiotics during the experimental period

- Auto-immune diseases with oral manifestations

- Systemic diseases with an impact on periodontal healing (e.g. diabetes)

Table 2. Demographic characteristics of the study population

\section{Non-periodontitis group $(n=11) \quad$ Periodontitis group $(n=20)$}

Age (years)

Mean $\pm S D$

$24.3 \pm 0.9$

$48.2 \pm 12.0$

Minimum-maximum

23-26

30-73

Gender (male/female)

$2 / 9$

$6 / 14$

Smoking status

Non-smoker/Smoker

$11 / 0$

$17 / 3$ 
Table 3. Mean surface area $\left(\mathrm{mm}^{2}\right) \pm S D$ of the abrasion lesions caused by 2 minutes of brushing measured at several time intervals for both groups (nonperiodontitis/periodontitis group). The percentage of area reduction per 24 hours' period was also calculated.

\begin{tabular}{lccccc} 
& \multicolumn{5}{c}{ Time interval } \\
\cline { 2 - 6 } & Oh & $24 \mathrm{~h}$ & $48 \mathrm{~h}$ & $72 \mathrm{~h}$ & $96 \mathrm{~h}$ \\
Non-perio group & & & & & \\
Mean $\pm S D\left(\right.$ in $\mathrm{mm}^{2}$ ) & $22.5 \pm 24.7$ & $12.3 \pm 12.3$ & $6.2 \pm 9.6$ & $8.4 \pm 12.6$ & $10.0 \pm 0.0$ \\
$\mathrm{~N}$ & 11 & 10 & 7 & 3 & 1 \\
Area reduction (in \%) & & 48.7 & 74.6 & 78.4 & 85.4 \\
Perio group & & & & & \\
Mean $\pm S D$ (in mm ${ }^{2}$ ) & $25.5 \pm 34.8$ & $18.3 \pm 24.8$ & $17.1 \pm 22.0$ & $12.6 \pm 13.0$ & $19.4 \pm 0.0$ \\
N & $15^{*}$ & $13^{*}$ & $6 *$ & $4^{*}$ & 1 \\
Area reduction (in \%) & & 45.4 & 76.4 & 71.6 & 1.65
\end{tabular}

$\mathrm{N}$ : The number of patients in whom the lesion is visible ${ }^{*}$ number $=$ number of smokers 
Table 4. Average surface area $\left(\mathrm{mm}^{2}\right) \pm S D$ of the abrasion lesions caused by 2 minutes, 1 minute and 30 seconds of brushing per day in the non-periodontitis group. The percentage of area reduction per 24 hour-period was also calculated.

\begin{tabular}{|c|c|c|c|c|c|c|c|}
\hline & Oh & $24 h$ & $48 h$ & $72 \mathrm{~h}$ & $96 h$ & $120 \mathrm{~h}$ & $240 \mathrm{~h}$ \\
\hline \multicolumn{8}{|l|}{$2 \min$} \\
\hline Mean $\pm S D\left(\right.$ in $\left.\mathrm{mm}^{2}\right)$ & $22.5 \pm 24.7$ & $12.3 \pm 12.3$ & $6.2 \pm 9.6$ & $8.4 \pm 12.6$ & $10.0 \pm 0.0$ & $6.2 \pm 0.0$ & $0.0 \pm 0.0$ \\
\hline $\mathrm{N}$ & 11 & 10 & 7 & 3 & 1 & 1 & 0 \\
\hline Patient $n^{\circ}$ & & (11) & $(7,8,10)$ & $(1,2, \underline{5}, 6)$ & $(\underline{3}, \underline{4})$ & & (9) \\
\hline Area reduction (in \%) & & 48.7 & 74.6 & 78.4 & 85.4 & 38.0 & 100.0 \\
\hline \multicolumn{8}{|l|}{$1 \mathrm{~min}$} \\
\hline Mean $\pm S D\left(\right.$ in $\left.\mathrm{mm}^{2}\right)$ & $7.4 \pm 9.0$ & $4.0 \pm 2.7$ & $0.5 \pm 0.0$ & $0.0 \pm 0.0$ & & & \\
\hline $\mathrm{N}$ & 6 & 5 & 1 & 0 & & & \\
\hline Patient $n^{\circ}$ & $(1,2,6,10,11)$ & (8) & $(\underline{3}, \underline{4}, \underline{5}, 7)$ & (9) & & & \\
\hline Area reduction (in \%) & & 43.1 & 98.4 & 100.0 & & & \\
\hline \multicolumn{8}{|l|}{$30 \mathrm{sec}$} \\
\hline Mean $\pm S D\left(\right.$ in $\left.\mathrm{mm}^{2}\right)$ & $1.4 \pm 0.9$ & $0.5 \pm 0.4$ & $0.0 \pm 0.0$ & & & & \\
\hline $\mathrm{N}$ & 5 & 2 & 0 & & & & \\
\hline Patient $n^{\circ}$ & $(1,6,7,8,10,11)$ & $(2, \underline{5}, \underline{9})$ & $(\underline{3}, \underline{4})$ & & & & \\
\hline Area reduction (in \%) & & 73.9 & 100.0 & & & & \\
\hline
\end{tabular}

$\mathrm{N}$ : The number of patients in whom a lesion was visible (between parenthesis: patient number for patients without lesions, or with already healed lesions. In case the patient had a thin biotype, this number is underlined) 
